\title{
Thyroid hormone analogs and metabolites: new applications for an old hormone?
}

\author{
P Reed Larsen
}

Considerable excitement was generated at the 79th Annual Meeting of the American Thyroid Association by developments in the use of naturally occurring iodothyronine metabolites as potential pharmacologic agents. In addition, new clinical data were presented on the effects of chemical thyroid hormone analogs for the treatment of hyperlipidemia.

The keynote speaker was Dr Thomas Scanlan from the Oregon Health and Science University, who reviewed the effects of the naturally occurring $T_{4}$ metabolite 3-iodothyronamine $\left(T_{1} A M\right)$, which is generated from $\mathrm{T}_{4}$ by decarboxylation of the alanine side chain and partial deiodination of the ring structures. Dr Scanlan and his colleagues have shown that both $\mathrm{T}_{1} \mathrm{AM}$ and its fully deiodinated derivative, $\mathrm{T}_{0} \mathrm{AM}$, have several acute and dramatic physiological effects, which are not at all what one might expect of $\mathrm{T}_{4}$ metabolites. $\mathrm{T}_{1} \mathrm{AM}$ is an agonist that can activate trace amine-associated receptor 1 , a G-protein-coupled receptor expressed in many different tissues, including the heart. $\mathrm{T}_{1} \mathrm{AM}$ can also bind to the $\alpha 2$ adrenergic receptor. Injection of $\mathrm{T}_{1} \mathrm{AM}$ into mice induces profound bradycardia and hypothermia within minutes, and leads to dose-dependent negative inotropic and chronotropic effects on the heart. These effects can be reproduced in isolated, perfused heart preparations. $\mathrm{T}_{1} \mathrm{AM}$ has been identified by liquid chromatography-tandem mass spectrometry in a number of mammalian tissues and fluids, including the central nervous system and serum.

A potential therapeutic application of $T_{1} A M$ or $\mathrm{T}_{0} \mathrm{AM}$ has been demonstrated in a mouse experimental model of stroke injury. Administration of either of these metabolites at pharmacological doses induces hypothermia and reduces infarct size when given either ....chemical

analogs

of thyroid

hormone can

be engineered

to create

a desired

effect, such as

suppression

of LDL

cholesterol

levels...

PR Larsen is

the Editor-in-

Chief of Nature

Clinical Practice

Endocrinology \&

Metabolism.

Competing interests

The author declared no

competing interests.

www.nature.com/clinicalpractice doi:10.1038/ncpendmet1025 shortly before or at the time of injury. This observation might be explained by the rapid decrease in oxygen consumption and body temperature that occurs in rodents given these compounds. Although these data are still preliminary, many additional developments seem likely in this rapidly advancing field, which aims to exploit the pharmacological applications of $\mathrm{T}_{1} \mathrm{AM}$ or other naturally occurring $\mathrm{T}_{4}$ metabolites.

New clinical data on the effects of thyroid hormone analogs that target thyroid hormone receptor $\beta$ in the liver were also reviewed by Dr Paul Ladenson of Johns Hopkins University School of Medicine. One agent, eprotirome (KB2115), was given to healthy volunteers for 2 weeks; no adverse effects on cardiac function were reported but treated individuals showed a significant decrease in total cholesterol and LDL cholesterol levels. This decrease occurred despite a small concurrent decrease in serum total $T_{4}$ and free $T_{4}$ levels, apparently as a result of modest feedback suppression of TSH by eprotirome. No weight loss or changes in metabolic rate were observed. These preliminary results suggest that chemical analogs of thyroid hormone can be engineered to create a desired effect, such as suppression of LDL cholesterol levels, without producing systemic thyrotoxicosis.

These interesting results with eprotirome and $T_{1} A M$ offer us unique insights into a fastmoving area of drug discovery. Designing synthetic thyroid hormone analogs that target specific receptors and/or tissues on the one hand, as well as exploring alternative mechanisms for the action of thyroid hormone and its metabolites on the other, could result in the discovery of novel classes of useful pharmacotherapeutic agents. 